\title{
Macrophage expression and prognostic significance of the long pentraxin PTX3 in COVID-19
}

\author{
Enrico Brunetta $\mathbb{1}^{1,2,10}$, Marco Folci ${ }^{1,2,10}$, Barbara Bottazzi ${ }^{1,10}$, Maria De Santis $\mathbb{D}^{1}$, Giuseppe Gritti $\mathbb{1}^{3}$, \\ Alessandro Protti ${ }^{1,2}$, Sarah N. Mapelli', Stefanos Bonovas ${ }^{1,2}$, Daniele Piovani ${ }^{1,2}$, Roberto Leone ${ }^{1}$, \\ Ilaria My ${ }^{1,2}$, Veronica Zanon', Gianmarco Spata ${ }^{1}{ }^{1}$, Monica Bacci', Domenico Supino², \\ Silvia Carnevale², Marina Sironi', Sadaf Davoudian ${ }^{1}{ }^{1}$, Clelia Peano ${ }^{114}$, Francesco Landi³, \\ Fabiano Di Marco ${ }^{5,6}$, Federico Raimondi ${ }^{5}$, Andrea Gianatti ${ }^{7}$, Claudio Angelini',

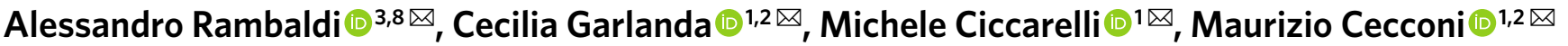 \\ and Alberto Mantovani 1 1,2,9凶
}

\begin{abstract}
Long pentraxin 3 (PTX3) is an essential component of humoral innate immunity, involved in resistance to selected pathogens and in the regulation of inflammation ${ }^{1-3}$. The present study was designed to assess the presence and significance of PTX3 in Coronavirus Disease 2019 (COVID-19) ${ }^{4-7}$. RNA-sequencing analysis of peripheral blood mononuclear cells, single-cell bioinformatics analysis and immunohistochemistry of lung autopsy samples revealed that myelomonocytic cells and endothelial cells express high levels of PTX3 in patients with COVID-19. Increased plasma concentrations of PTX3 were detected in 96 patients with COVID-19. PTX3 emerged as a strong independent predictor of 28-d mortality in multivariable analysis, better than conventional markers of inflammation, in hospitalized patients with COVID-19. The prognostic significance of PTX3 abundance for mortality was confirmed in a second independent cohort (54 patients). Thus, circulating and lung myelomonocytic cells and endothelial cells are a major source of PTX3, and PTX3 plasma concentration can serve as an independent strong prognostic indicator of short-term mortality in COVID-19.
\end{abstract}

Highly pathogenic betacoronaviruses causing severe acute respiratory syndrome, Middle East respiratory syndrome and the current COVID-19 pandemic, affect the lower respiratory tract leading to critical acute respiratory distress syndrome (ARDS) and fatality in a high percentage of cases $^{4-7}$. Severe acute respiratory syndrome coronavirus 2 (SARS-CoV-2) infection is characterized by variable clinical forms, with symptoms including fever, cough and general malaise in mild and moderate cases, which progress to severe pneumonia or ARDS, shock and/or multiple organ failure, requiring admission to intensive care units (ICUs) in more severe cases. The high morbidity and mortality observed in the COVID-19 pandemic are caused by alveolar damage and pneumonia, cardiovascular complications and multi-organ failure.

SARS-CoV-2 interacts with angiotensin-converting enzyme 2 expressed by pneumocytes in the alveolar lining, leading to lung injury. Angiotensin-converting enzyme 2 is also widely expressed on endothelial cells, thus possibly explaining the evidence of direct viral infection of the endothelium, diffuse endothelial inflammation and widespread microvascular dysfunction, leading to organ ischemia, inflammation, edema and a procoagulant state ${ }^{7-12}$. In addition, uncontrolled activation of innate and adaptive immunity in response to the infection results in hyperinflammatory responses, which, by affecting lung tissue and blood vessels, contribute to ARDS pathogenesis, shock and multi-organ failure ${ }^{7,13}$. Macrophages are central drivers of uncontrolled inflammation and tissue damage associated with COVID-19 (refs. ${ }^{7,13,14}$ ).

PTX3 is a key component of humoral innate immunity, belonging to the family of pentraxins ${ }^{1,2}$. In contrast with its relative, the short pentraxin C-reactive protein (CRP), essentially produced by the liver in response to interleukin (IL)- 6 during the acute-phase response ${ }^{3}$, PTX3 is rapidly produced by several cell types, including myeloid cells, endothelial cells and respiratory epithelial cells, particularly in response to IL-1, tumor necrosis factor, microbial molecules and tissue damage ${ }^{1,2}$. PTX3 is an essential component of humoral innate immunity, involved in resistance to selected pathogens and in the regulation of inflammation ${ }^{1,2}$. The similarity with CRP prompted investigations as to the usefulness of PTX3 as a marker in diverse human conditions of infective or inflammatory origin. The local production by different cell types at inflammatory sites and the release of the preformed protein by neutrophils in response to primary proinflammatory cytokines or microbial recognition accounts for the rapidity of PTX3 increase in these

\footnotetext{
'Humanitas Clinical and Research Center-IRCCS, Milan, Italy. ${ }^{2}$ Department of Biomedical Sciences, Humanitas University, Milan, Italy. ${ }^{3}$ Unit of Hematology, Azienda Ospedaliera Socio Sanitaria Territoriale Papa Giovanni XXIII, Bergamo, Italy. ${ }^{4}$ Institute of Genetic and Biomedical Research, UoS Milan, National Research Council, Milan, Italy. ${ }^{5}$ Unit of Pneumology, Azienda Ospedaliera Socio Sanitaria Territoriale Papa Giovanni XXIII, Bergamo, Italy. ${ }^{6}$ Department of Health Sciences, University of Milan, Milan, Italy. ${ }^{7}$ Unit of Pathology, Azienda Ospedaliera Socio Sanitaria Territoriale Papa Giovanni XXIII, Bergamo, Italy. ${ }^{8}$ Department of Oncology \& Hemato-Oncology, University of Milan, Milan, Italy. ${ }^{9}$ The William Harvey Research Institute, Queen Mary University of London, London, UK. ${ }^{10}$ These authors contributed equally: Enrico Brunetta, Marco Folci, Barbara Bottazzi. ${ }^{\mathrm{e}_{\mathrm{e}}-\mathrm{mail}}$ : arambaldi@asst-pg23.it; cecilia.garlanda@humanitasresearch.it; michele.ciccarelli@humanitas.it; maurizio.cecconi@humanitas.it; alberto.mantovani@humanitasresearch.it
} 
conditions. Increased PTX3 plasma concentrations were described in infections of fungal, bacterial and viral origin ${ }^{15,16}$, severe inflammatory response syndrome, sepsis ${ }^{17-20}$ and cardiovascular diseases ${ }^{21}$. In different pathological conditions, high PTX3 plasma concentrations were associated with disease severity and mortality ${ }^{17-19}$. Moreover, PTX3 has been shown to serve as a biomarker of disease activity in inflammatory conditions involving the vascular bed, ranging from atherosclerosis to vasculitis ${ }^{21-24}$.

Previous findings on the prognostic significance of PTX 3 in systemic inflammatory conditions, as well as in vascular pathology $y^{17,21}$ prompted the present investigation, which was designed to investigate the cellular sources and significance of PTX3 in patients with COVID-19.

We conducted an in silico bioinformatic analysis of the expression of PTX3 using public databases ${ }^{25-27}$. SARS-CoV-2 strongly induced or amplified PTX3 transcript expression in two lines representative of respiratory tract epithelial cells, Calu-3 and A549 (Fig. 1a). The same trend was observed in normal human bronchial cells, although it did not reach statistical significance (GSE147507) ${ }^{25}$. Bulk RNA sequencing (RNA-seq) of purified monocytes (Fig. 1b) and bioinformatic analysis at single-cell level of peripheral blood mononuclear cells (PBMCs) obtained from patients with COVID19 (Fig. 1c) revealed that PTX3 was selectively expressed by COVID-19 neutrophils and monocytes (GSE150728) ${ }^{26}$. Moreover, bioinformatic analysis of COVID-19 bronchoalveolar lavage fluid (BALF) cells at single-cell level ${ }^{27}$ revealed that PTX3 was strongly expressed in neutrophils and monocyte-derived macrophage populations, as identified by molecular signatures (Fig. 1d).

Further confirmation of PTX 3 protein expression in patients with COVID-19 was obtained by immunohistochemistry on autopsy samples from the lungs of individuals with a confirmed positivity to SARS-CoV-2. An example of typical staining on lung specimens is shown in Fig. 2a, which revealed PTX3 positivity in alveolar macrophages, as well as in the adventitia of small vessels and in type II pneumocytes. PTX3 immunostaining was, in particular, observed in multinucleated macrophages (Fig. 2a). Interestingly, PTX3 staining was particularly intense in the adventitia and endothelium of small vessels (for example, the vessel occluded by a thrombus in Fig. 2b). Thus, bulk scRNA-seq analysis and immunohistology revealed that, in peripheral blood and lungs of patients with COVID-19, myelomonocytic cells express high amounts of PTX3. Moreover, immunohistology suggested that vascular cells, key players in disease pathogenesis, also express PTX3.

Based on previous studies on the prognostic ability of PTX3 in inflammatory conditions and on transcript expression mainly in myelomonocytic cells, which play a key role in pathogenesis, we assessed PTX3 plasma concentrations in 96 patients with COVID-19 consecutively admitted to Humanitas Clinical and Research Center. Demographic, clinical and laboratory features of patients are shown in Supplementary Table 1. In the overall population, patients had elevated PTX3 (median 17.3 $\mathrm{ng} \mathrm{m}^{-1}$, interquartile range (IQR) $10.1-39.8 \mathrm{ng} \mathrm{ml}^{-1}$ ), CRP (median $12.3 \mathrm{mg} \mathrm{dl}^{-1}, \mathrm{IQR} 6.2-22.3 \mathrm{mg} \mathrm{dl}^{-1}$ ) and IL-6 (median $55 \mathrm{pg} \mathrm{ml}^{-1}$; IQR $24-118 \mathrm{pg} \mathrm{ml}^{-1}$ ) concentrations. Similarly to our previous study ${ }^{28}$, we also observed elevated levels of ferritin (median $622 \mu \mathrm{g} \mathrm{ml}^{-1}$, IQR $182-987 \mu \mathrm{g} \mathrm{ml}^{-1}$ ), D-dimer (median $637 \mu \mathrm{g} \mathrm{ml}^{-1}$, IQR $409-1,394 \mu \mathrm{g} \mathrm{ml}^{-1}$ ) and lactate dehydrogenase (LDH; median $345 \mathrm{U} \mathrm{l}^{-1}$, IQR $268-431 \mathrm{U} \mathrm{l}^{-1}$ ). A reduction in lymphocytes (median $0.8 \times 10^{3}$ per $\mathrm{ml}$, IQR $0.6-1.1 \times 10^{3}$ per $\mathrm{ml}$ ) and eosinophils (median $0 \times 10^{3}$ per ml, IQR $0-0.1 \times 10^{3}$ per $\mathrm{ml}$ ) was also found, as previously reported ${ }^{28}$.

In the first cohort, 52 patients $(54.2 \%)$ were transferred to the ICU within $7 \mathrm{~d}$ from admission because of clinical worsening. The primary end point of death occurred in 22 patients (23\%), comprising 14 patients who died in ICU and 8 who died in medical wards, while a total of 58 patients $(60.4 \%)$ had been discharged alive and 16 patients (16.6\%) were still hospitalized on the data cutoff date. PTX3 values were higher in patients who finally died as compared with patients who survived (median $39.8 \mathrm{ng} \mathrm{ml}^{-1}$, IQR 20.2-75.7 $\mathrm{ng} \mathrm{ml}^{-1}$ versus $15.3 \mathrm{ng} \mathrm{ml}^{-1}$, IQR $8.2-21.3 \mathrm{ng} \mathrm{ml}^{-1}$, respectively; Fig. 2c). Moreover, higher PTX3 levels were found in ICU patients compared to ward patients (median $21.0 \mathrm{ng} \mathrm{ml}^{-1}$, IQR 15.6-46.3 $\mathrm{ng} \mathrm{ml}^{-1}$ versus median $12.4 \mathrm{ng} \mathrm{ml}^{-1}$, IQR 6.12-20.2 $\mathrm{ng} \mathrm{ml}^{-1}$, respectively; Fig. 2d).

After defining three PTX3 groups by tertiles (low-level group: $1.6-9.53 \mathrm{ng} \mathrm{ml} \mathrm{m}^{-1}$; medium-level group: 9.69-20.18 $\mathrm{ng} \mathrm{ml}^{-1}$; high-level group: $\left.20.61-147 \mathrm{ng} \mathrm{ml}^{-1}\right)$, the Kaplan-Meier analysis showed an overall 28 -d event-free survival of 0.88 (95\% confidence interval (CI) $0.71-0.95)$ in the medium-level group and $0.59(95 \%$ CI $0.42-0.72$ ) in the high-level group. No deaths were observed in the low-level group (log-rank test, $P=0.0001$; Fig. 2 e).

In the univariable Cox regression analysis, $\mathrm{PTX} 3$ was a strong predictor of mortality in patients with COVID-19 (crude hazard ratio (HR) 22.4; 95\% CI 5.8-86.2; $P<0.0001$ ). Based on the available literature and statistical considerations, we adjusted for potential confounding factors (age, ICU stay, days between symptom onset and PTX3 test, and the sequential organ failure assessment (SOFA) score at admission). PTX3 remained a strong predictor of 28-d mortality (adjusted HR (aHR) 27.6; 95\% CI 5.3-142.8; $P<0.0001$; Table 1). The proportional hazard assumption was not violated $(P=0.16)$. The ability of PTX3 to predict death in patients with COVID-19 was excellent in this cohort of 96 patients, either alone (Harrell's C-statistic $0.81 ; 95 \%$ CI $0.71-0.90$ ) or in the multivariable model (Harrell's C-statistic 0.87; 95\% CI 0.80-0.94).

Correlations between PTX3 and other inflammatory markers were assessed with the Spearman's test (Supplementary Table 2). PTX3 was significantly correlated with CRP, procalcitonin (PCT), IL-6, ferritin and D-dimer, but also with other poor COVID-19 prognostic factors, such as LDH, troponin-I, lymphocyte count and platelet count. The latter observation is consistent with previous findings in sepsis ${ }^{17}$. On the contrary, no correlation was observed between circulating concentrations of PTX3 and CXCL10, recently suggested as classifiers of severity in patients with COVID-19, but not considering death as the end point ${ }^{29}$ (Extended Data Fig. 1). Levels of PTX3, CRP, ferritin, IL-6 and D-dimer were also analyzed as continuous variables for predicting mortality (univariable Cox regressions; Table 2). IL-6 was also significantly, but weakly, associated with mortality in the unadjusted analysis.

To validate the findings regarding the prognostic significance for mortality of PTX3 in patients with COVID-19, we analyzed a second, independent cohort of 54 patients from Azienda Socio Sanitaria Territoriale (ASST) Papa Giovanni XXIII Hospital, Bergamo (Table 3). There were considerable differences between the two cohorts (Supplementary Table 3). These differences largely reflect the 'frontline' dramatic situation of the Bergamo institution in February through April 2020. Despite the different demographic and clinical characteristics between the two cohorts (Supplementary Table 3), the prognostic power of PTX3 was confirmed (crude HR 6.9, 95\% CI 1.25-38.2, $P=0.026$; and Harrell's C-statistic 0.71, 95\% CI 0.58-0.84). Also in this second cohort, PTX3 was a better prognostic factor for death than CRP and IL-6 (Table 3). Thus, results obtained in two independent cohorts indicate that PTX3 blood concentrations represent a strong prognostic indicator of short-term mortality in COVID-19, better related to outcome than conventional biomarkers including IL-6.

As of 15 September 2020, the number of COVID-19 cases was reported to be more than 25 million, leading to more than 900,000 deaths worldwide (https://www.who.int/docs/default-source/coron aviruse/20200630-covid-19-sitrep-162.pdf?sfvrsn=e00a5466_2/). Elevated levels of CRP, cytokine and chemokines ${ }^{28,30}$, together with low lymphocyte and eosinophil counts, characterize patients with severe disease ${ }^{31}$.

Moreover, as discussed below, in depth profiling of immunity has provided new candidate tools $\mathrm{s}^{29,32-36}$. However, a reliable biomarker 

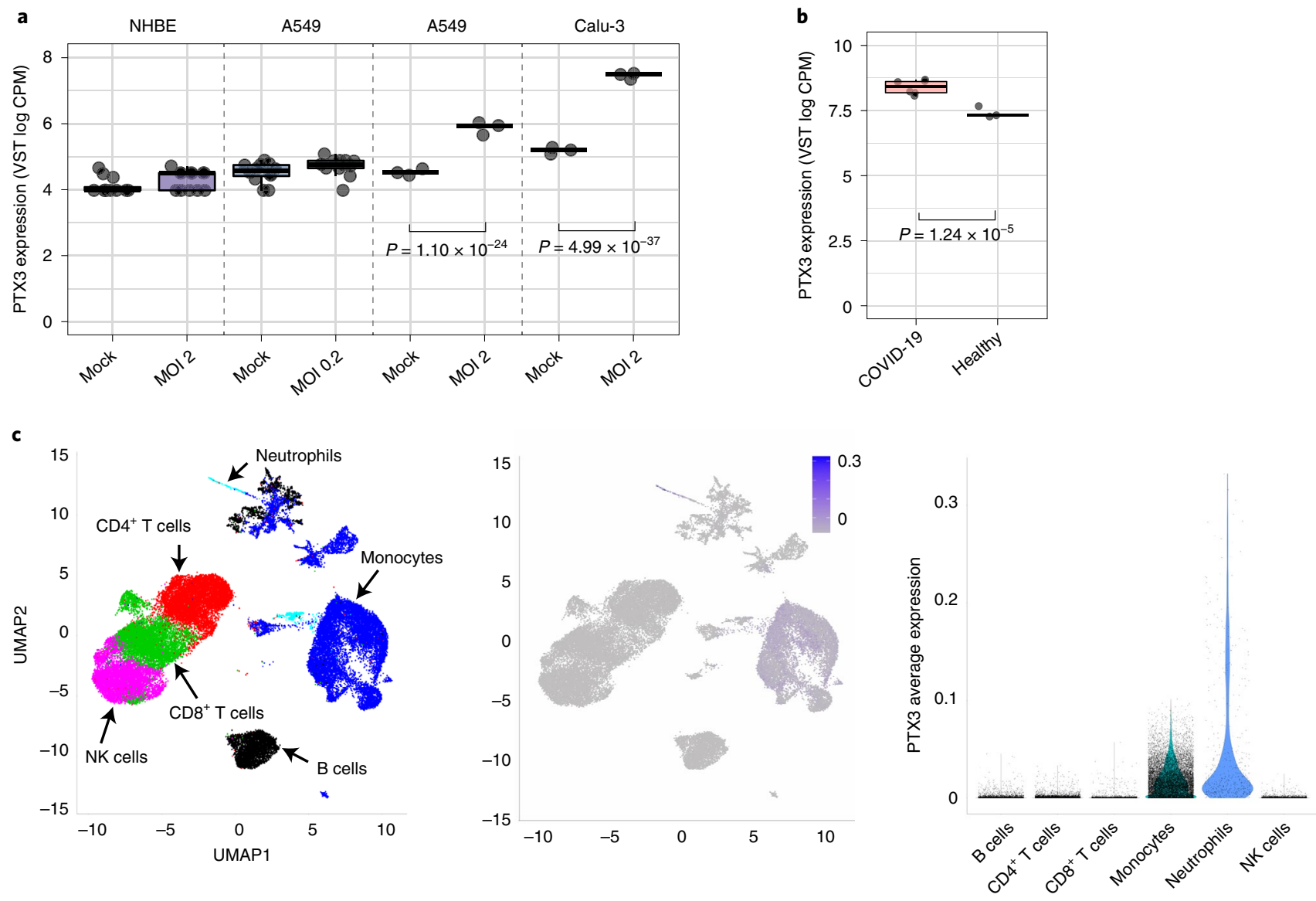

d
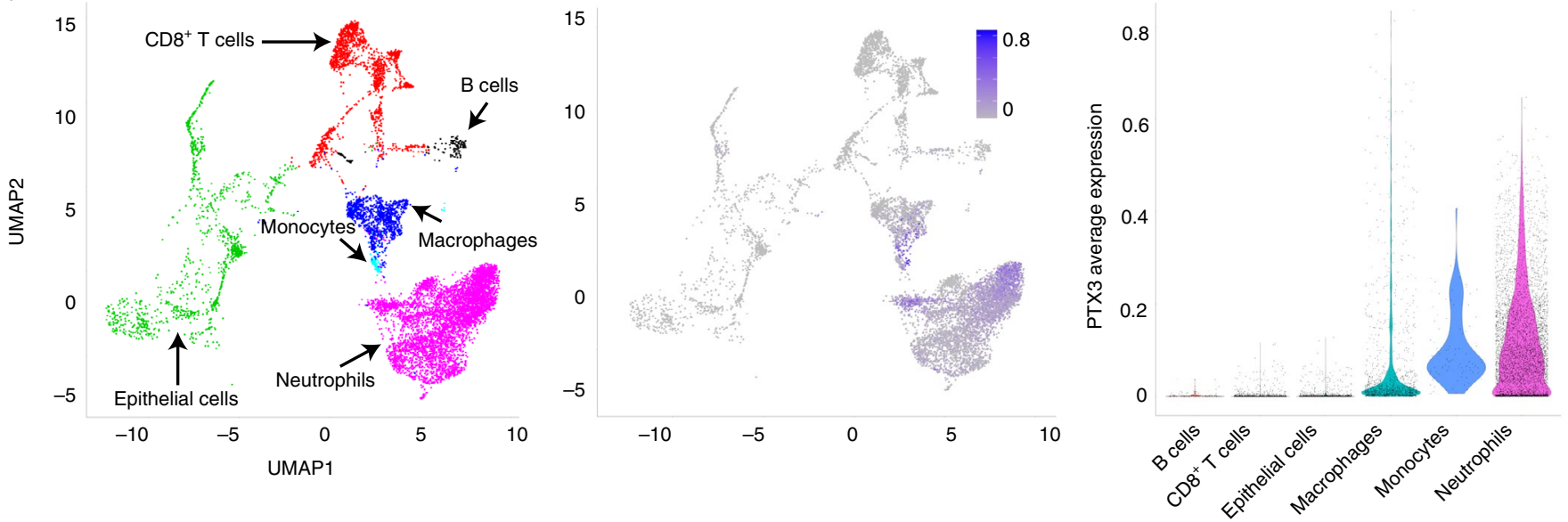

Fig. 1 | In silico analysis of PTX3 expression in SARS-CoV-2-infected cells and patients with COVID-19. a, PTX3 levels of in vitro SARS-CoV-2-infected respiratory epithelial cells: normal human bronchial epithelial cells (NHBCs; at a multiplicity of infection (MOI) of $2, n=12$ independent biological replicates) and human lung cancer cell lines A549 (MOI: 0.2, $n=12$ independent biological replicates; MOI: $2, n=3$ independent biological replicates) and Calu-3 (MOI: 2, n=3 independent biological replicates). Comparisons are with mock-treated controls, and significant changes in PTX3 expression are indicated. CPM, counts per million; VST, variance-stabilization transformation $\mathbf{b}$, PTX3 expression measured in RNA-seq of peripheral monocytes from patients hospitalized with COVID-19 $(n=6)$ and healthy donors $(n=3)$. In $\mathbf{a}$ and $\mathbf{b}$, box plots represent the median (center line), upper and lower quartiles (box limits), 1.5 times the IQR (whiskers) and outliers (elements outside boxes). P values were calculated using a two-sided Student's $t$-test and were adjusted for multiple comparisons with Bonferroni correction. c, Single-cell RNA-seq (scRNA-seq) of COVID-19 PBMCs. Left, uniform manifold approximation and projection (UMAP) showing Seurat-guided clustering of PBMC populations. Each point represents a single-cell colored according to cluster designation. NK, natural killer cells. Center, heat map showing the expression levels of PTX3. Right, violin plots representing expression and distribution of PTX3 within predicted PBMC populations. d, sCRNA-seq of COVID-19 BALF. Left, UMAP showing Seurat-guided clustering of BALF populations. Center, heat map showing the expression levels of PTX3. Right, violin plots representing expression and distribution of PTX3 within predicted BALF populations. Single-cell expression values are shown after imputation. 
a

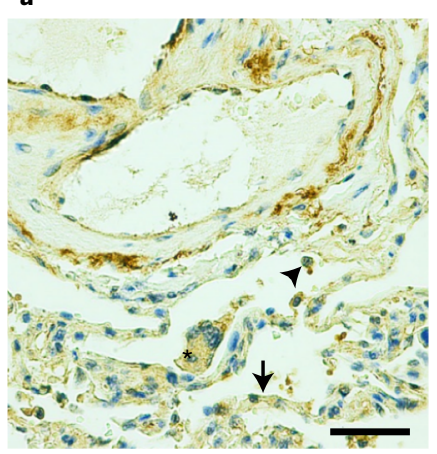

c

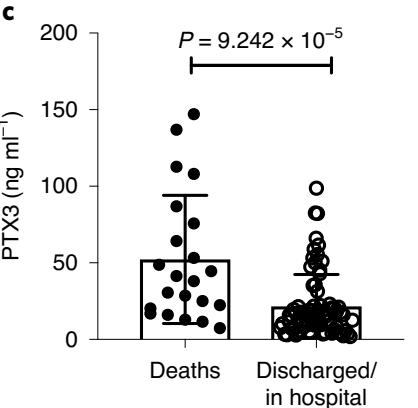

e

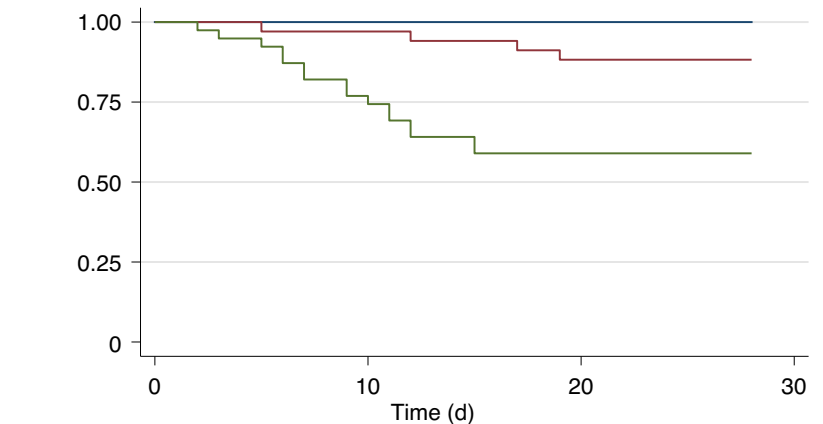

Number at risk

PTX3 low 23

PTX3 medium 34

PTX3 high 39

(0)

(1)

$(1)$
$(9)$

23
33

33
30

$(0)$
$(3)$
$(7)$

23

(0)

(0)

(0)

0

- PTX3 low-level group

- PTX3 high-level group

- PTX3 medium-level group

Fig. 2 | PTX3 expression and prognostic value in patients with COVID-19. a,b, PTX3 immunolocalization in COVID-19 lung specimens. a, PTX3 in type II pneumocytes (arrow) and alveolar macrophages (arrowhead). Note the granular cytoplasmic positivity in a multinuclear macrophage, denoted by the asterisk (immunoperoxidase stain; $\times 40$ magnification; scale bar, $50 \mu \mathrm{m}$ ). b, PTX3 in the adventitia (arrowhead) and endothelium (arrow) of a small vessel occluded by a thrombus (immunoperoxidase stain; $\times 20$ magnification; scale bar, $50 \mu \mathrm{m}$ ). Images are representative of one of four SARS-CoV-2-positive patients analyzed. c,d, PTX3 plasma concentration in 96 patients with COVID-19 admitted to Humanitas Clinical and Research Hospital. c, Patients were divided based on the primary outcome (mortality; deaths, $n=22$, discharged/in hospital patients, $n=74)$. $\mathbf{d}$, PTX3 plasma concentration in patients admitted to medical wards $(n=50)$ or ICU $(n=46)$. Individual data points are shown with the mean \pm standard deviation (s.d.). In $\mathbf{c}$ and $\mathbf{d}, P$ values were calculated using a two-tailed, unpaired Wilcoxon rank-sum test, without adjustments. e, Kaplan-Meier curves by level of PTX3 (low, medium and high) defined by tertiles. No events were observed in the low-level group. The 28 - $d$ event-free survival was 0.88 ( $95 \% \mathrm{Cl} 0.71-0.95$ ) in the medium-level group and 0.59 (95\% Cl 0.42-0.72) in the high-level group. Two-sided log-rank test, $P=0.0001$.
Table 1 | PTX3 as predictor of death in hospitalized patients with COVID-19

\begin{tabular}{llll} 
Variables & aHR & $\mathbf{9 5 \% ~ C l}$ & P value \\
\hline $\begin{array}{l}\text { PTX3 (per 1 ng ml-1 } \\
\text { increase) }\end{array}$ & 27.6 & $(5.3-142.8)$ & $<0.0001$ \\
\hline Age (per 1-year increase) & 1.12 & $(1.04-1.20)$ & 0.005 \\
\hline ICU (yes or no) & 6.19 & $(0.94-40.88)$ & 0.058 \\
\hline SOFA (per 1-point increase) & 0.99 & $(0.81-1.23)$ & 0.970 \\
$\begin{array}{l}\text { Days onset to PTX3 (per 1-d } \\
\text { increase) }\end{array}$ & 0.99 & $(0.87-1.12)$ & 0.887 \\
\hline
\end{tabular}

Multivariable Cox regression analysis on 96 patients from Humanitas Clinical and Research Hospital adjusted for age, stay in ICU, SOFA score at admission and days between onset of

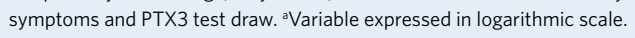

of poor outcome in COVID-19 is still lacking. The early and accurate triaging of the patients may contribute to better patient management and stratification in clinical trials. The present study was designed to investigate expression and clinical significance of the fluid-phase pattern-recognition receptor PTX3 in COVID-19. We found that PTX3 was induced by SARS-CoV-2 in respiratory tract epithelial cells. In patients with COVID-19, PTX3 analyzed in bulk and at single-cell level was selectively expressed by monocytes among circulating cells and by lung macrophages, as well as by endothelial cells by immunohistochemistry. High PTX3 plasma levels were a strong independent indicator of short-term 28-d mortality. In this patient cohort, PTX3 fared substantially better than other known prognostic markers, including CRP, IL-6, ferritin and D-dimer.

PTX3 blood levels above the normal value $\left(<2 \mathrm{ng} \mathrm{ml}^{-1}\right)$ can be found in the subclinical inflammatory status of cardiovascular diseases $^{37}$, as well as in infections and sepsis, with increasing median values when moving to more severe conditions. In a large study conducted in 1,326 unselected hospitalized individuals (14\% with infectious diseases), PTX3 levels above the 95th percentile of healthy non-hospitalized individuals $\left(>6.4 \mathrm{ng} \mathrm{ml}^{-1}\right)$ were significantly associated with higher mortality in the short term, independently of

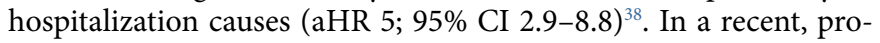
spective, observational study including 547 ICU patients $(42.4 \%$ with infections), PTX3 serum levels were independently associated with 28-d mortality when adjusted for age, gender, chronic diseases and immunosuppression (HR 1.87; 95\% CI 1.41-2.48) ${ }^{39}$. In another recent study conducted on 281 patients with sepsis, serum PTX3 > $26 \mathrm{ng} \mathrm{ml}^{-1}$ was associated with mortality ${ }^{40}$. The sample size of the original and validation cohorts investigated in the present study is limited (96 and 54 patients, respectively); therefore, we analyzed PTX3 as a continuous predictor and considered that, at this stage, it would have been inappropriate to define a threshold value, which would require an order of magnitude larger cohort. However, with this caveat, previous results and our results suggest that circulating PTX3 levels tenfold above the normal value reflect a severe systemic inflammatory involvement with an ominous outcome.

PTX3 is produced by diverse cell types including myelomonocytic cells, lung epithelial cells and endothelial cells. In the present study, we found that SARS-CoV-2 induced gene expression of PTX3 in respiratory tract epithelial cells. Using bulk RNA-seq and bioinformatic analysis at single-cell level, we found that PTX3 was selectively expressed by monocytes among circulating leukocytes. Moreover, in lung BALF, single-cell analysis on public databases revealed selective expression of PTX3 in neutrophils and macrophages (Fig. 1d). Myeloid cells are credited to play a major role in the pathogenesis of the disease ${ }^{6,7,13,14}$.

PTX3 was originally cloned in endothelial cells ${ }^{1}$ and vascular cells are a major source of this component of humoral innate 
Table 2 | Inflammatory and other biomarkers as predictors of death in patients hospitalized with COVID-19

\begin{tabular}{|c|c|c|c|}
\hline Variables & HR & $95 \% \mathrm{Cl}$ & $P$ value \\
\hline $\begin{array}{l}\text { PTX3 (per } 1 \mathrm{ng} \mathrm{ml}^{-1} \\
\text { increase) }\end{array}$ & 22.4 & $(5.8-86.2)$ & $<0.0001$ \\
\hline CRP (per $1 \mathrm{mg} \mathrm{dl}^{-1}$ increase) & 1.03 & $(0.99-1.07)$ & 0.082 \\
\hline IL-6 (per $1 \mathrm{pg} \mathrm{ml}^{-1}$ increase) $)^{\mathrm{a}}$ & 3.70 & $(1.27-10.83)$ & 0.017 \\
\hline PCT $\left(\leq 1\right.$ versus $\left.>1 \mathrm{mg} \mathrm{dl}^{-1}\right)$ & 0.86 & $(0.31-2.4)$ & 0.780 \\
\hline $\begin{array}{l}\text { Ferritin (per } 1 \mathrm{ng} \mathrm{ml}^{-1} \\
\text { increase) }\end{array}$ & 4.17 & $(0.85-20.5)$ & 0.079 \\
\hline $\begin{array}{l}\text { D-dimer (per } 1 \mu \mathrm{g} \mathrm{l^{-1 }} \\
\text { increase) }^{\mathrm{a}}\end{array}$ & 2.3 & $(0.82-6.54)$ & 0.113 \\
\hline
\end{tabular}

Univariable Cox analysis of 96 patients from Humanitas Clinical and Research Hospital. ${ }^{2}$ Variable expressed in logarithmic scale.

Table 3 | PTX3 as a predictor of death in a validation cohort of hospitalized patients with COVID-19

\begin{tabular}{|c|c|c|c|}
\hline Variables & HR & $95 \% \mathrm{Cl}$ & $P$ value \\
\hline PTX3 (per $1 \mathrm{ng} \mathrm{ml}^{-1}$ increase) ${ }^{a}$ & 6.9 & $(1.25-38.2)$ & 0.026 \\
\hline CRP (per $1 \mathrm{mg} \mathrm{dl}^{-1}$ increase) & 1.04 & $(0.97-1.10)$ & 0.203 \\
\hline IL-6 (per 1 pg ml ${ }^{-1}$ increase) $)^{a}$ & 2.14 & $(0.86-5.29)$ & 0.099 \\
\hline
\end{tabular}

Univariable Cox analysis of 54 patients from ASST Papa Giovanni XIII Hospital. ${ }^{\circledR}$ Variable expressed in logarithmic scale.

immunity, although their role could not be directly ascertained in the present study. Endothelial cells and the lung vascular bed have emerged as major determinants of COVID-19-associated microvascular thrombosis and disease pathogenesis ${ }^{7-12}$. PTX3 plasma levels have been shown to correlate with the severity of disease in various forms of vascular pathology, including small-vessel vasculitis, coronary heart disease and Kawasaki disease ${ }^{24,37,41}$. The latter observation raises the issue of its significance in the Kawasaki-like disease, now called multisystem inflammatory syndrome in children (MIS-C), observed in children after COVID-19 (refs. ${ }^{42-45}$ ). Of interest, our data show a significant correlation between PTX3 and D-dimer, a surrogate of coagulation cascade activation and a marker of venous thrombosis, and between PTX3 and troponin-I, a marker of myocardial disease; both myocardial inflammation and acute ischemic heart disease have been described in COVID-19 (refs. ${ }^{46,47}$ ). These observations raise the possibility that the strong prognostic significance of PTX3 in COVID-19 may reflect its positioning at the very intersection between macrophage-driven inflammation and vascular involvement.

PTX3 is a fluid-phase pattern-recognition molecule that binds selected viruses and plays a role in antimicrobial immunity ${ }^{1,2}$. Moreover, PTX3 has a regulatory role in inflammation by interfering with selectin-dependent neutrophil recruitment and by regulating the complement cascade ${ }^{48,49}$. Uncontrolled complement activation has been shown to play an important role in COVID-19 disease pathogenesis and to represent a therapeutic target ${ }^{50}$. It is tempting to speculate that high levels of PTX3 in COVID-19 reflect failed negative regulation of uncontrolled inflammation. The actual role of PTX3 and, more generally, of humoral innate immunity in resistance against SARS-CoV-2 and in disease pathogenesis deserves further investigation.

An earlier and more accurate triaging may improve patient management and stratification in clinical trials. A number of recent studies ${ }^{29,32-35}$ have recently reported extensive deep profiling of immune responses in COVID-19 and signatures relevant as disease classifiers or prognostic indicators. In the present study, we found that in two independent patient cohorts, early in disease progression, PTX3 was a strong prognostic indicator of short-term death, a hard end point, better than other markers such as IL-6 and CRP. IL-6, IL-10 and CXCL10 (IP-10) were recently suggested to serve as classifiers of patients with COVID-19, with end points other than death $^{29}$. In 20 patients from our original cohort, we found no correlation between PTX3 and CXCL10 (Extended Data Fig. 1), possibly suggesting distinct spectra of clinical significance in relation to end points. We speculate that the strong prognostic significance of PTX3 for short-term mortality reflects its production by myeloid cells and endothelial cells in the lungs. With the caveat of the limited number of patients in our two independent cohorts, the results reported here suggest that PTX3 may serve as an early low-cost/low-tech biomarker for patient management, follow-up and stratification in clinical trials. Large studies are needed to assess whether PTX3 can represent a low-cost complementary or alternative approach to sophisticated immune signatures in patient management, assessment of response to different therapies and clinical trial design.

\section{Online content}

Any methods, additional references, Nature Research reporting summaries, source data, extended data, supplementary information, acknowledgements, peer review information; details of author contributions and competing interests; and statements of data and code availability are available at https://doi.org/10.1038/ s41590-020-00832-x.

Received: 3 July 2020; Accepted: 29 October 2020;

Published online: 18 November 2020

\section{References}

1. Garlanda, C., Bottazzi, B., Magrini, E., Inforzato, A. \& Mantovani, A. PTX3, a humoral pattern recognition molecule, in innate immunity, tissue repair and cancer. Physiol. Rev. 98, 623-639 (2018).

2. Bottazzi, B., Doni, A., Garlanda, C. \& Mantovani, A. An integrated view of humoral innate immunity: pentraxins as a paradigm. Annu. Rev. Immunol. 28, 157-183 (2010).

3. Pepys, M. B. The pentraxins 1975-2018: serendipity, diagnostics and drugs. Front. Immunol. 9, 2382 (2018).

4. Wu, F. et al. A new coronavirus associated with human respiratory disease in China. Nature 579, 265-269 (2020).

5. Zhu, N. et al. A novel coronavirus from patients with pneumonia in China, 2019. N. Engl. J. Med. 382, 727-733 (2020).

6. Fauci, A. S., Lane, H. C. \& Redfield, R. R. COVID-19-navigating the uncharted. N. Engl. J. Med. 382, 1268-1269 (2020).

7. Cecconi, M., Forni, G. \& Mantovani, A. Ten things we learned about COVID-19. Intensive Care Med. 46, 1590-1593 (2020).

8. Ackermann, M. et al. Pulmonary vascular endothelialitis, thrombosis and angiogenesis in COVID-19. N. Engl. J. Med. 383, 120-128 (2020).

9. Varga, Z. et al. Endothelial cell infection and endotheliitis in COVID-19. Lancet 395, 1417-1418 (2020).

10. Goshua, G. et al. Endotheliopathy in COVID-19-associated coagulopathy: evidence from a single-centre, cross-sectional study. Lancet Haematol. 7, e575-e582 (2020)

11. Libby, P. \& Luscher, T. COVID-19 is, in the end, an endothelial disease. Eur. Heart J. 41, 3038-3044 (2020).

12. Rambaldi, A. et al. Endothelial injury and thrombotic microangiopathy in COVID-19: treatment with the lectin-pathway inhibitor narsoplimab. Immunobiology https://doi.org/10.1016/j.imbio.2020.152001 (2020).

13. Wang, J., Jiang, M., Chen, X. \& Montaner, L. J. Cytokine storm and leukocyte changes in mild versus severe SARS-CoV-2 infection: review of 3,939 COVID-19 patients in China and emerging pathogenesis and therapy concepts. J. Leukoc. Biol. 108, 17-41 (2020).

14. Merad, M. \& Martin, J. C. Pathological inflammation in patients with COVID-19: a key role for monocytes and macrophages. Nat. Rev. Immunol. 20, 355-362 (2020).

15. Cunha, C. et al. Genetic PTX3 deficiency and aspergillosis in stem-cell transplantation. N. Engl. J. Med. 370, 421-432 (2014).

16. Sprong, T. et al. Pentraxin 3 and C-reactive protein in severe meningococcal disease. Shock 31, 28-32 (2009).

17. Caironi, P. et al. Pentraxin 3 in patients with severe sepsis or shock: the ALBIOS trial. Eur. J. Clin. Invest. 47, 73-83 (2017). 
18. Muller, B. et al. Circulating levels of the long pentraxin PTX3 correlate with severity of infection in critically ill patients. Crit. Care Med. 29, 1404-1407 (2001).

19. Mauri, T. et al. Persisting high levels of plasma pentraxin 3 over the first days after severe sepsis and septic shock onset are associated with mortality. Intensive Care Med. 36, 621-629 (2010).

20. Lee, Y. T. et al. Pentraxin-3 as a marker of sepsis severity and predictor of mortality outcomes: a systematic review and meta-analysis. J. Infect. 76, $1-10$ (2018)

21. Jenny, N. S., Arnold, A. M., Kuller, L. H., Tracy, R. P. \& Psaty, B. M. Associations of pentraxin 3 with cardiovascular disease and all-cause death: the Cardiovascular Health Study. Arterioscler. Thromb. Vasc. Biol. 29, 594-599 (2009).

22. Fazzini, F. et al. PTX3 in small-vessel vasculitides: an independent indicator of disease activity produced at sites of inflammation. Arthritis Rheum. 44, 2841-2850 (2001).

23. van Rossum, A. P. et al. Abundance of the long pentraxin PTX3 at sites of leukocytoclastic lesions in patients with small-vessel vasculitis. Arthritis Rheum. 54, 986-991 (2006).

24. Ramirez, G. A. et al. PTX3 intercepts vascular inflammation in systemic immune-mediated diseases. Front Immunol. 10, 1135 (2019).

25. Blanco-Melo, D. et al. Imbalanced host response to SARS-CoV-2 drives development of COVID-19. Cell 181, 1036-1045 (2020).

26. Wilk, A. J. et al. A single-cell atlas of the peripheral immune response in patients with severe COVID-19. Nat. Med. 26, 1070-1076 (2020).

27. Chua, R. L. et al. COVID-19 severity correlates with airway epitheliumimmune cell interactions identified by single-cell analysis. Nat. Biotechnol. 38, 970-979 (2020).

28. Cecconi, M. et al. Early predictors of clinical deterioration in a cohort of 239 patients hospitalized for COVID-19 infection in Lombardy, Italy. J. Clin. Med. 9, 1548 (2020).

29. Laing, A. G. et al. A dynamic COVID-19 immune signature includes associations with poor prognosis. Nat. Med. 26, 1623-1635 (2020).

30. Hou, H. et al. Using IL-2R/lymphocytes for predicting the clinical progression of patients with COVID-19. Clin. Exp. Immunol. 201, 76-84 (2020).

31. Chen, R. et al. Longitudinal hematologic and immunologic variations associated with the progression of COVID-19 patients in China. J. Allergy Clin. Immunol. 146, 89-100 (2020).

32. Mathew, D. et al. Deep immune profiling of COVID-19 patients reveals distinct immunotypes with therapeutic implications. Science 369, eabc8511 (2020).

33. Arunachalam, P. S. et al. Systems biological assessment of immunity to mild versus severe COVID-19 infection in humans. Science 369, 1210-1220 (2020).

34. Hadjadj, J. et al. Impaired type I interferon activity and inflammatory responses in severe COVID-19 patients. Science 369, 718-724 (2020).
35. Kuri-Cervantes, L. et al. Comprehensive mapping of immune perturbations associated with severe COVID-19. Sci. Immunol. 5, eabd7114 (2020).

36. Zhu, L. et al. Single-cell sequencing of peripheral mononuclear cells reveals distinct immune response landscapes of COVID-19 and influenza patients. Immunity 53, 685-696 (2020).

37. Ristagno, G. et al. Pentraxin 3 in cardiovascular disease. Front Immunol. 10, 823 (2019).

38. Bastrup-Birk, S. et al. Pentraxin-3 level at admission is a strong predictor of short-term mortality in a community-based hospital setting. J. Intern. Med. 277, 562-572 (2015).

39. Hansen, C. B. et al. Complement-related pattern recognition molecules as markers of short-term mortality in intensive care patients. J. Infect. 80, 378-387 (2020)

40. Song, J. et al. Biomarker combination and SOFA score for the prediction of mortality in sepsis and septic shock: a prospective observational study according to the Sepsis-3 definitions. Medicine 99, e20495 (2020).

41. Katsube, Y. et al. PTX3, a new biomarker for vasculitis, predicts intravenous immunoglobulin unresponsiveness in patients with Kawasaki disease. J. Am. Coll. Cardiol. 57, E2038 (2011).

42. Viner, R. M. \& Whittaker, E. Kawasaki-like disease: emerging complication during the COVID-19 pandemic. Lancet 395, 1741-1743 (2020).

43. Whittaker, E. et al. Clinical characteristics of 58 children with a pediatric inflammatory multisystem syndrome temporally associated with SARS-CoV-2. JAMA 324, 259-269 (2020).

44. Mantovani, A. \& Netea, M. G. Trained innate immunity, epigenetics and COVID-19. N. Engl. J. Med. 383, 1078-1080 (2020).

45. Verdoni, L. et al. An outbreak of severe Kawasaki-like disease at the Italian epicentre of the SARS-CoV-2 epidemic: an observational cohort study. Lancet 395, 1771-1778 (2020).

46. Escher, F. et al. Detection of viral SARS-CoV-2 genomes and histopathological changes in endomyocardial biopsies. ESC Heart Fail. 7, 2440-2447 (2020).

47. Lodigiani, C. et al. Venous and arterial thromboembolic complications in COVID-19 patients admitted to an academic hospital in Milan, Italy. Thromb. Res. 191, 9-14 (2020).

48. Deban, L. et al. Regulation of leukocyte recruitment by the long pentraxin PTX3. Nat. Immunol. 11, 328-334 (2010).

49. Deban, L. et al. Binding of the long pentraxin PTX3 to factor H: interacting domains and function in the regulation of complement activation. J. Immunol. 181, 8433-8440 (2008).

50. Risitano, A. M. et al. Complement as a target in COVID-19? Nat. Rev. Immunol. 20, 343-344 (2020).

Publisher's note Springer Nature remains neutral with regard to jurisdictional claims in published maps and institutional affiliations.

(c) The Author(s), under exclusive licence to Springer Nature America, Inc. 2020 


\section{Methods}

Study design and participants. The study was approved by the Humanitas Clinica and Research Center Ethical Committee (authorization 233/20); the requirement for informed consent was waived. This study analyzed a cohort of 96 patients. We included all males and nonpregnant females, 18 years of age or older, who were admitted to Humanitas Clinical and Research Center (Milan, Italy) between 04 March and 09 May 2020 (data cutoff: 13 May) with a laboratory-confirmed diagnosis of COVID-19. Hospital admission criteria were based on a positive RT-PCR assay for SARS-CoV-2 associated with respiratory failure requiring oxygen therapy, or radiological evidence of substantial pulmonary infiltrates on chest computed tomography (CT) scan, or reduction in respiratory/cardiopulmonary reserve as assessed by a 6-min walking test, or due to frailty related with patient comorbidity. The primary outcome of the study was death. Fifty-two patients of 96 (54\%) were transferred to ICUs because of the requirement for invasive ventilation or noninvasive mechanical ventilation with an oxygen fraction of over $60 \%$. Patients with continuous positive airway pressure therapy were followed up by the ICU outreach team and ward physicians in COVID-19 wards. ARDS was defined according to the Berlin definition ${ }^{51}$

A second, independent cohort of 54 patients admitted to the ASST Papa Giovanni XXIII, Bergamo, Italy, from 22 February to 04 April 2020, with a laboratory-confirmed diagnosis of COVID-19, was analyzed to confirm our results. In this cohort, the same criteria of admission as the first cohort were adopted.

Laboratory test, demographic and medical history. Laboratory testing at hospital admission included: complete blood count, renal and liver function (transaminase, total/direct/indirect bilirubin, gamma-glutamyl transferase and alkaline phosphatase), creatinine kinase, LDH, myocardial enzymes, electrolytes and triglycerides. A panel of acute-phase reactants including IL-6, serum ferritin, D-dimer, CRP, fibrinogen and PCT, and CXCL10/IP-10, was performed. IL-6 and CXCL10 were measured using a custom-designed plate on an ELLA automated immunoassay system (Bio-Techne) according to the manufacturer's instructions. Body temperature, blood pressure, heart rate, peripheral saturation and respiratory rate were measured in all patients. A chest CT scan and arterial blood gas analysis were performed in the emergency department. In all patients, PTX3 was measured within the first few days after the admission date $(2.1 \pm 1.6 \mathrm{~d})$. Pneumococcal and Legionella urinary antigen tests were routinely performed. Nasopharyngeal swabs for influenza A, B and H1N1 were also routinely performed to exclude coinfections. Additional microbiological tests were performed to exclude other pathogens as possible etiological agents when suggested by clinical conditions (bacterial cultures of sputum, blood and urine). We obtained a comprehensive present and past medical history from patients. Positivity was assessed on the basis of RT-PCR for SARS-CoV-2 on a respiratory tract sample tested by our laboratory, in accordance with the protocol established by the WHO (World Health Organization; https://www.who.int/emergencies/diseases/novel-coronavirus-2019/ technical-guidance/laboratory-guidance/) or by commercially available methods (GeneFinder COVID-19 Plus RealAmp Kit ELIThech Group) and Allplex 2019-nCoV Assay (Seegene, Arrow Diagnostics). Due to the high false-negative rate of RT-PCR from pharyngeal swabs, each patient was tested with two different swabs to increase the detection rate ${ }^{52}$. In cases of negative assays in throat-swab specimens, but with suggestive clinical manifestations, presence of contact history or suggestive radiological evidence for COVID-19, the detection was performed on BALF or endotracheal aspirate, which has higher diagnostic accuracy. All study data, including demographics, medical history and laboratory test results, were extracted from electronic medical records and checked by a team of three expert physicians.

Purification of human peripheral blood monocytes. For RNA expression analysis, monocytes were isolated from peripheral blood samples of healthy donors and patients with COVID-19. Monocytes were enriched from Ficoll-isolated PBMCs by positive selection using a CD14 Microbeads kit (Miltenyi Biotec), according to the manufacturer's instructions. Purity of monocytes was $>89 \%$. The resulting cells were processed for RNA extraction.

FACS analysis. Purity of monocytes was determined by FACS analysis. Cell viability was determined by Aqua LIVE/Dead Fixable-405-nm staining (Invitrogen). Cells were then incubated with an Fc receptor blocking reagent, human (Miltenyi Biotec). The following human antibodies and their dilutions were used: anti-CD45-BUV805 (clone HI30, BD Biosciences, no. 612891; 1:40), anti-CD11b-BV786 (clone IRCF44, BioLegend, no. 301346; 1:40), anti-CD3-BUV737 (clone UCHT1, BD Biosciences, no. 564308; 1:80), anti-CD4-PECy5 (clone OKT4, BioLegend, no. 317412; 1:40), anti-CD8-BUV496 (clone RPA-T8, BD Biosciences, no. 612942; 1:40), anti-CD56-PECF594 (clone NCAM16.2, BD Biosciences, no. 564849; 1:20), anti-CD19-APCH7 (clone HIB19, BD Biosciences, no. 560727; 1:40), anti-CD14-AF488 (clone M5E2, BD Biosciences, no. 557700; 1:50), anti-CD16-BUV563 (clone 3G8, BD Biosciences, no. 748851; 1:20), anti-CD66b-APCR700 (clone G10F5, BioLegend, no. 305114; 1:20) and anti-HLA-DR-BV480 (clone G46-6, BD Biosciences, no. 566113; 1:40). Cells were analyzed on a FACSymphony (BD Bioscience). Data were analyzed with FlowJo software (version 9.6.9; Treestar). The gating strategy is reported in Extended Data Fig. 2.

Total RNA sequencing. RNA was purified with the Direct-zol RNA Microprep or Miniprep Kit (Zymosan), according to the manufacturer's instructions. RNA quality control was performed with the 4200 Tape Station system (Agilent). Total RNA-seq library preparation was performed starting from 1 ng of total RNA with the SMART-Seq Stranded Kit (Clontech-Takara). Libraries obtained were qualitatively assessed by using TapeStation 4200 (Agilent) and quantified by Qubit Fluorimeter (Thermo Fisher Scientific). Afterwards, they were multiplexed in equimolar pools and sequenced on a NextSeq-550 Illumina Platform generating at least 80 million 75-bp paired-end reads per sample.

Bioinformatic analysis. Data relative to the transcriptional response to SARS-CoV-2 infection were either obtained by sequencing peripheral monocytes of hospitalized patients (compared to healthy controls) or derived from datasets deposited within the Gene Expression Omnibus (GEO). Data relative to bulk transcription in normal human bronchial and malignant cell lines (Calu-3 and A549) after SARS-CoV-2 infection were derived from the experiments within the series GSE147507 (ref. ${ }^{25}$ ) using SRA-Tools (version 2.10.0). Bulk RNA-seq raw reads of sorted monocytes, derived from six patients with COVID-19 and three healthy donors, were obtained after demultiplexing with 'bcl2fastq2' Conversion Software (version 2.19.0). Raw bulk RNA-seq reads were quality inspected with the software 'FastQC' (https://www.bioinformatics.babraham.ac.uk/projects/fastqc/ version 0.11.9) and aligned with STAR (version 2.6.1) ${ }^{53}$ on the GRCh38 genome guided by GENCODE annotation (version 33).

Gene summarized counts were processed in $\mathrm{R}$ (version 3.5.2). Base bioinformatic functions for Bioconductor packages were allowed by the Biobase package (version 2.42.0). Genes whose expression was greater than two reads were filtered and VST normalized with the R package DESeq2 (version 1.22.2) ${ }^{54}$. Significantly changing genes after SARS-CoV-2 infection were obtained with DESeq2 and limma (version 3.38.3). Gene annotation and conversions among gene identifiers were performed with the R packages 'org.Hs.eg.db' (version 3.7.0) and AnnotationDBi (version 1.44.0). A similar workflow was adopted to align, quantify and compare bulk RNA-seq of in vitro infected cell lines, confronting each infected cell type with the respective mock-treated counterpart. Gene identifier conversions were performed with the org.Hs.eg.db library (https://www.bioconductor.org/ packages//2.10/data/annotation/html/org.Hs.eg.db.html). Plots were rendered with the R library 'ggplot2' (version 3.3.2; https://ggplot2.tidyverse.org/).

Available scRNA-seq experiments, related to the BALF of SARS-CoV-2 individuals, were obtained from the public repositories GEO and FigShare platform under the identifiers GSE145926 (refs. ${ }^{55,56}$ ) and the FigShare platform (https://figshare.com/articles/COVID-19_severity_correlates_with_ airway_epithelium-immune_cell_interactions_identified_by_single-cell_ analysis/12436517), respectively. scRNA-seq datasets of SARS-CoV-2-infected PBMCs (deposited in GEO under GSE150728) ${ }^{26}$ were explored with the portal cellxgene (https://chanzuckerberg.github.io/cellxgene/) and obtained from the COVID-19 Cell Atlas portal (https://www.covid19cellatlas.org/\#wilk20/). Sparse count matrices or Seurat objects were obtained as released and processed in the $\mathrm{R}$ environment 3.6.1 with the Seurat package ${ }^{57}$ (version 3.1.5) and confirmed with the published pipelines shared by respective authors.

Classification of clusters, defined according to the authors' parameters, was performed with 'SingleR' package (version 1.0.1), matching the Blueprint reference annotation. The distribution of PTX3 expression was obtained after imputation with the 'Rmagic' package (version 2.0.3, based on reticulate version 1.16) and mapped on confidently defined populations ${ }^{58}$.

Sample collection and PTX3 measurement. Venous blood samples were collected during the first $5 \mathrm{~d}$ after hospital admission (mean \pm s.d., $2.1 \pm 1.6 \mathrm{~d}$ ), centrifuged, and EDTA plasma was stored at $-80^{\circ} \mathrm{C}$ until use. PTX3 plasma concentrations were measured, as previously described ${ }^{59}$, by a sandwich ELISA (detection limit $0.1 \mathrm{ng} \mathrm{ml} \mathrm{m}^{-1}$, inter-assay variability from $8-10 \%$ ) developed in-house, by personnel who were blinded to patient characteristics. Briefly, the sandwich ELISA is based on the rat monoclonal antibody MNB4, used as capturing antibody, and on biotin-labeled rabbit IgG anti-human PTX3 in detection. Rabbit IgG were purified by immunoaffinity on PTX3-conjugated Sepharose-CNBr columns. Both MNB4 and rabbit IgG were obtained by immunizing a rat and rabbit, respectively, with purified recombinant PTX3 and were selected based on reactivity towards human recombinant PTX3 in ELISA and SDS-PAGE. No cross reaction was observed towards human CRP. Measurements were taken from distinct samples, and each sample was tested in duplicate. In each analytical session, a sample obtained from a pool of plasma from healthy donors was used as an internal control. The mean PTX3 concentration measured in this sample was $1.88 \pm 0.6 \mathrm{ng} \mathrm{ml}^{-1}$. For the Bergamo cohort, blood samples were collected from February 22 through April 42020.

Immunohistochemistry. Immunohistochemical staining was performed as follows: 3 - $\mu \mathrm{m}$-thick sections were prepared from formalin-fixed paraffin-embedded autoptic tissue lung blocks from a patient with COVID-19 and 
were dried at $60^{\circ} \mathrm{C}$ for $20 \mathrm{~min}$. The sections were placed in a BOND-III Automated Immunohistochemistry Vision Biosystem (Leica Microsystems) according to the following protocol. First, tissues were deparaffinized and pretreated with the Epitope Retrieval Solution 1 (citrate buffer; $\mathrm{pH}$ 5.9-6.1) at $100^{\circ} \mathrm{C}$ for $10 \mathrm{~min}$. After washing, peroxidase blocking was carried out for $5 \mathrm{~min}$ using the Bond Polymer Refine Detection Kit DC9800 (Leica Microsystems). Tissues were again washed and then incubated with the primary antibody (affinity-purified rabbit IgG anti-human PTX3, final concentration $\left.2-\mu \mathrm{g} \mathrm{ml}^{-1}\right)^{60}$ for $15 \mathrm{~min}$. Subsequently, tissues were incubated with polymer for $8 \mathrm{~min}$ and developed with DAB-Chromogen for $10 \mathrm{~min}$. The optimal concentration of the primary antibody and the best antigenic unmasking to identify the PTX3 protein were established on ten histological sections from different paraffin blocks of SARS-CoV-2-positive patients.

Statistical methods. Demographic, clinical, laboratory and outcome data were obtained from electronic medical records and patient chart notes using a standardized data collection form. Descriptive statistics included means with s.d. values and medians with IQRs for continuous variables, and frequency analyses (percentages) for categorical variables. Wilcoxon rank-sum tests (for continuous variables) and Fisher's exact tests (for categorical variables) were applied using $\mathrm{R}$ environment, $\mathrm{R}$ base package (version 1.1.463 2009-2018 RStudio and version 3.6.1). Data analysis and data visualization were obtained with STATA/IC 16.1 (revision 05 Mar 2020; StataCorp). Linearity of continuous variables was checked by comparing models with the linear term to the model with restricted cubic splines. Because of nonlinearity, PTX3, ferritin, D-dimer and IL-6 were used in the logarithmic scale for regression analysis. PCT and CRP were used in binary form, based on laboratory cutoff values. Correlation between variables was evaluated with Spearman's rank correlation coefficient (rho). To identify the association between PTX3 concentrations and the outcome in patients hospitalized with COVID-19, we used time-to-event (survival) methods for censored observations. The study end point was 'death' within $28 \mathrm{~d}$ from hospital admission. Time to event was defined as the time from hospital admission until the date of event or censoring. Patients discharged early and alive from the hospital were considered event-free through day 28 (ref. ${ }^{61}$ ). The data cutoff was 13 May 2020. Kaplan-Meier estimates were used to draw the cumulative incidence curves by level of PTX3 defined by tertiles (low, medium and high); finally, they were compared with a log-rank test. Furthermore, multivariable Cox proportional hazards models of prognostic factors were used. The analyses were based on non-missing data (missing data not imputed). Confounders were selected according to a review of the literature, statistical relevance and consensus opinion by an expert group of physicians and methodologists. Gender has been excluded because of its irrelevance in modifying the HR in the analysis (HR modification was $<10 \%$ when gender was included in the PTX3 univariable Cox model). After fitting the model, the proportional hazards assumption was examined on the basis of Schoenfeld residuals. The HRs are presented with their $95 \%$ CIs and the respective $P$ values. A ratio higher than 1.0 implies a higher probability of death compared to the reference group.

To validate our findings regarding the prognostic significance of PTX3 in relation to mortality in patients with COVID-19, we analyzed a second, independent cohort of 54 patients from the ASST Papa Giovanni XXIII, Bergamo. To measure the predictive power in both cohorts, we computed the Harrell's $\mathrm{C}$-statistic with its $95 \% \mathrm{CI}^{62}$.

Reporting Summary. Further information on research design is available in the Nature Research Reporting Summary linked to this article.

\section{Data availability}

The RNA-seq data used for Fig. 1b are publicly available within the GEO under accession GSE160351. RNA-seq and scRNA-seq data derived from previously published studies were obtained from the GEO, with accession codes GSE147507 and GSE145926. A Seurat object relative to scRNA-seq was obtained from the FigShare platform: https://figshare.com/articles/COVID-19 severity correlates with_airway_epithelium-immune_cell_interactions_identified_by_single-cell_ analysis/12436517). The clinical data that support the findings of this study are available in Supplementary Dataset 1 and from the corresponding authors upon request. Source data are provided with this paper.

\section{Code availability}

All code used in this work is consistent with the 'best practice workflows' suggested by the developers of each used tool (Methods). RNA-seq data from different experiments, including the in-house generated sequencing experiments, were analyzed with the same pipeline, based on the described software. scRNA-seq experiments, derived from publicly available datasets, were instead processed with the codes defined by the authors to reach consistent cell clustering to be screened for the gene of our interest. Cluster annotation, gene imputation and plots were performed with standard parameters for each of the described tools. The list of custom scripts generated to obtain the results shown in Fig. 1 is available within FigShare: https://figshare.com/articles/software/Macrophage_expression_and_
prognostic_significance_of_the_long_pentraxin_PTX3_in_COVID-19_Custom_ scripts_and_analyses/13140248.

\section{References}

51. Force, A. D. T. et al. Acute respiratory distress syndrome: the Berlin definition. JAMA 307, 2526-2533 (2012).

52. Alhazzani, W. et al. Surviving sepsis campaign: guidelines on the management of critically ill adults with coronavirus disease 2019 (COVID-19). Crit. Care Med. 48, e440-e469 (2020).

53. Dobin, A. et al. STAR: ultrafast universal RNA-seq aligner. Bioinformatics 29, 15-21 (2013).

54. Love, M. I., Huber, W. \& Anders, S. Moderated estimation of fold change and dispersion for RNA-seq data with DESeq2. Genome Biol. 15, 550 (2014).

55. Bost, P. et al. Host-viral infection maps reveal signatures of severe COVID-19 patients. Cell 181, 475-1488 (2020).

56. Liao, M. et al. Single-cell landscape of bronchoalveolar immune cells in patients with COVID-19. Nat. Med. 26, 842-844 (2020).

57. Butler, A., Hoffman, P., Smibert, P., Papalexi, E. \& Satija, R. Integrating single-cell transcriptomic data across different conditions, technologies and species. Nat. Biotechnol. 36, 411-420 (2018).

58. van Dijk, D. et al. Recovering gene interactions from single-cell data using data diffusion. Cell 174, 716-729 (2018).

59. Knoflach, M. et al. Pentraxin-3 as a marker of advanced atherosclerosis results from the Bruneck, ARMY and ARFY Studies. PLoS ONE 7, e31474 (2012)

60. Locatelli, M. et al. The long pentraxin PTX3 as a correlate of cancer-related inflammation and prognosis of malignancy in gliomas. J. Neuroimmunol. 260, 99-106 (2013).

61. Harrell, F. E. Jr., Lee, K. L. \& Mark, D. B. Multivariable prognostic models: issues in developing models, evaluating assumptions and adequacy, and measuring and reducing errors. Stat. Med. 15, 361-387 (1996).

62. Uno, H., Cai, T., Pencina, M. J., D’Agostino, R. B. \& Wei, L. J. On the $\mathrm{C}$-statistics for evaluating overall adequacy of risk prediction procedures with censored survival data. Stat. Med. 30, 1105-1117 (2011).

\section{Acknowledgements}

This work was supported by institutional funds, by a donation from the Dolce \& Gabbana fashion house (to A.M. and C.G.), by a grant from Ministero della Salute for COVID-19 (to A.M. and C.G.) and by the European Research Council (ERC project PHII-669415 to A.M.). S.D. was supported by the European Sepsis Academy/Innovative Training Networks (ESA/ITN) from the European Commission (H2020-MSCA-ITN-2015; grant no. 676129). We acknowledge CINECA and ELIXIR for the availability of high performance computing resources and support. This work was conducted in the framework of, and made possible by, the collective effort of the Humanitas COVID-19 Task Force (the members are listed in the Supplementary Note).

\section{Author contributions}

E.B., M.F., B.B, M.D.S., A.P. and C.G. contributed to the experimental design, collected patient data, performed data interpretation and drafted the manuscript. S.N.M., S.B. and D.P. performed the bioinformatic and statistical analysis. A.G. performed the histological evaluation. R.L., M.S. and S.D. conducted experiments. I.M. and C.A. collected patient data. V.Z., G.S. and M.B. collected specimens. D.S., S.C. and C.P. collected specimens and contributed to RNA-seq analysis. F.L., F.D.M., F.R. and G.G. collected patient data and performed data interpretation for the validation cohort. A.R., M. Cicarelli. and M. Cecconi. contributed to the experimental design and supervision of the study. A.M. conceived the study and finalized the manuscript. All authors approved the final manuscript.

\section{Competing interests}

A.M., C.G. and B.B. are inventors of a patent (EP20182181) on PTX3 and obtain royalties on related reagents. The other authors declare no competing interests.

\section{Additional information}

Extended data is available for this paper at https://doi.org/10.1038/s41590-020-00832-x.

Supplementary information is available for this paper at https://doi.org/10.1038/ s41590-020-00832-x.

Correspondence and requests for materials should be addressed to A.R., C.G., M.C., M.C. or A.M

Peer review information Nature Immunology thanks William Wu, Peter Garred, and the other, anonymous, reviewer(s) for their contribution to the peer review of this work. L. A. Dempsey was the primary editor on this article and managed its editorial process and peer review in collaboration with the rest of the editorial team.

Reprints and permissions information is available at www.nature.com/reprints. 


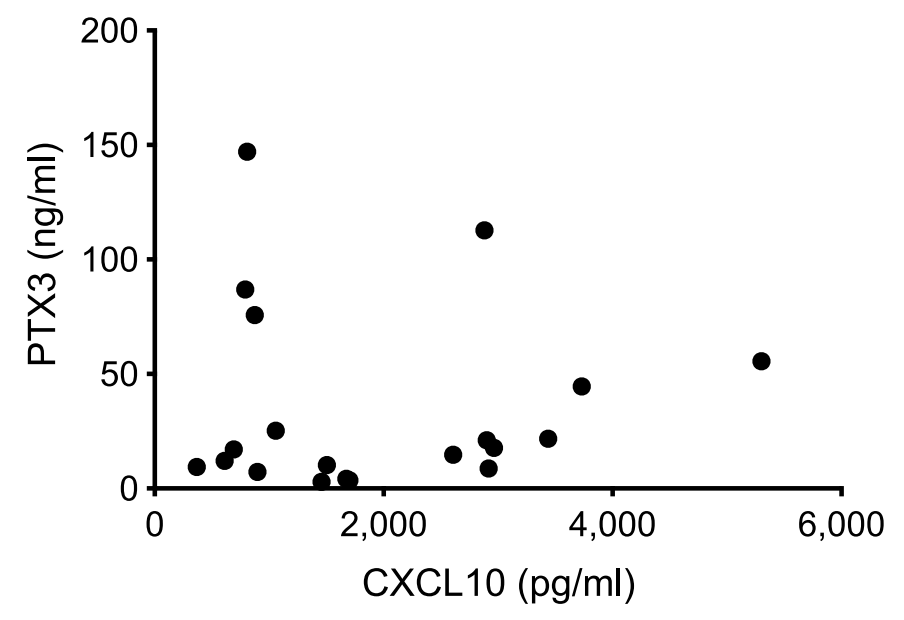

Extended Data Fig. 1 | Lack of correlation between PTX3 and CXCL10. Analysis of PTX3 and CXCL10 levels was performed in a subgroup of COVID patients $(n=20)$. Spearman's correlation coefficient $\rho=0.099 ; p=0.677$. 


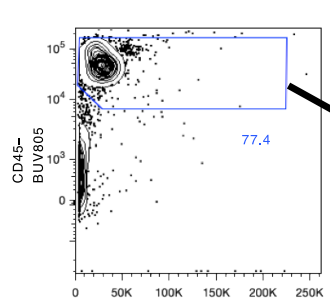

ssc-

A

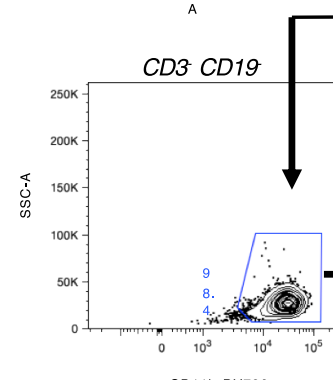

CD11b-BV786
All Cells

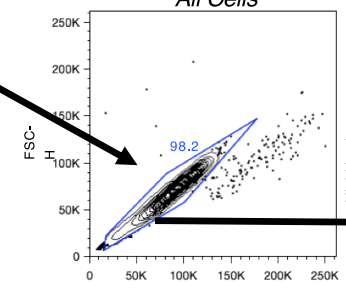

FSC-

A

$C D 11 b$

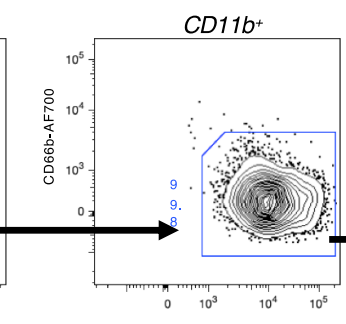

HLA-DR-BV480
Single Cells

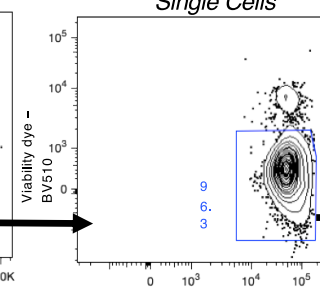

CD45-BUV805

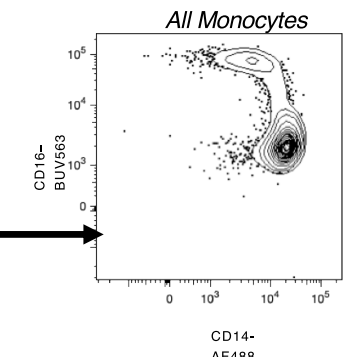

Extended Data Fig. 2 | Monocyte gating strategy. The gating strategy was used to verify purity of monocytes used to generate RNA-seq data of Fig. 1b. CD $45^{+}$cells were gated based on CD45 expression and on the SSC-A morphological parameter to exclude debris. Then, single cells were selected on the basis of FSC-A and FSC-H. Dead cells were further excluded through a viability dye. Monocytes were selected as CD11b high CD3- CD19- cells. 


\section{Reporting Summary}

Nature Research wishes to improve the reproducibility of the work that we publish. This form provides structure for consistency and transparency in reporting. For further information on Nature Research policies, see our Editorial Policies and the Editorial Policy Checklist.

\section{Statistics}

For all statistical analyses, confirm that the following items are present in the figure legend, table legend, main text, or Methods section.

$\mathrm{n} / \mathrm{a} \mid$ Confirmed

$\bigotimes$ The exact sample size $(n)$ for each experimental group/condition, given as a discrete number and unit of measurement

$\square$ \ A statement on whether measurements were taken from distinct samples or whether the same sample was measured repeatedly

The statistical test(s) used AND whether they are one- or two-sided

Only common tests should be described solely by name; describe more complex techniques in the Methods section.

$\square$ A description of all covariates tested

$\square \bigotimes$ A description of any assumptions or corrections, such as tests of normality and adjustment for multiple comparisons

$\square$ A full description of the statistical parameters including central tendency (e.g. means) or other basic estimates (e.g. regression coefficient)

$\triangle$ AND variation (e.g. standard deviation) or associated estimates of uncertainty (e.g. confidence intervals)

$X$ For null hypothesis testing, the test statistic (e.g. $F, t, r$ ) with confidence intervals, effect sizes, degrees of freedom and $P$ value noted

Give $P$ values as exact values whenever suitable.

Х $\square$ For Bayesian analysis, information on the choice of priors and Markov chain Monte Carlo settings

Х $\square$ For hierarchical and complex designs, identification of the appropriate level for tests and full reporting of outcomes

\ $\square$ Estimates of effect sizes (e.g. Cohen's $d$, Pearson's $r$ ), indicating how they were calculated

Our web collection on statistics for biologists contains articles on many of the points above.

\section{Software and code}

Policy information about availability of computer code

Data collection Available dataset deposited in GEO and SRA Archive were retrived with the software SRA-Tools (version 2.10.0).

Preprocessed files were retrieved from source different platforms by http or ftp server connections.

Clinical data:

R Studio, Version 1.1.463 - (c) 2009-2018 RStudio, Inc.

Data analysis Genomic anaysis were based on GRCh38 genome and GENCODE annotation (version 33).

RNA-Seq analysis:

To generate raw sequencing reads files, the software bcl2fastq, version 2.19.0 was used.

Quality of reads was checked with fastqc, version 0.11.9.

Alignments and quantifications were performed with STAR-2.6.1.

Statistical comparisons, normalizations and plots were generated within $\mathrm{R}$ version 3.5.2

with the packages

Biobase_2.42.0 (Base functions for Bioconductor packages)

org.Hs.eg.db_3.7.0, AnnotationDbi_1.44.0 (Gene annotations and conversions)

limma_3.38.3 (contrast matrix design), DESeq2_1.22.2 (Normalization, differential expression)

ggplot2_3.3.2 (plots generation and saving in vectorial format)

Single cell RNA-Seq analysis:

Analyses were performed within R version 3.6.1

with the packages

Seurat_3.1.5 (Analysis, and exploration of single cell RNA sequencing data)

SingleR_1.0.1 (Cluster annotation based on reference data) 
reticulate_1.16 (Interface to 'Python' modules, classes, and functions) Rmagic_2.0.3 (Markov affinity-based graph imputation of cells)

Clinical data:

The software environment R (version 1.1.463 2009-2018 RStudio, Inc.) was used for data and life management. Data analysis and data visualization were obtained with STATA/IC 16.1 Revision 05 Mar 2020. Copyright 1985-2019 StataCorp LLC.

FACS analysis:

Data were analyzed with FlowJo software (version 9.6.9) (Treestar).

For manuscripts utilizing custom algorithms or software that are central to the research but not yet described in published literature, software must be made available to editors and

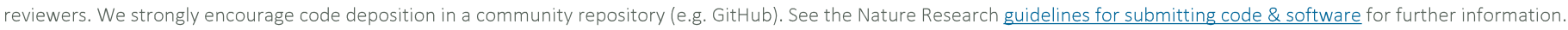

Data

Policy information about availability of data

All manuscripts must include a data availability statement. This statement should provide the following information, where applicable:

- Accession codes, unique identifiers, or web links for publicly available datasets

- A list of figures that have associated raw data

- A description of any restrictions on data availability

Data availability

The RNA-Seq data used for Figure $1 \mathrm{~b}$ are publicly available within the Gene Expression Omnibus (GEO) under the accession code GSE160351. RNA-Seq and single cell RNA-Seq data derived from previously published studies were obtained from public repositories: GSE147507 and GSE145926 series from GEO. A Seurat object relative to single cell RNA-Seq was obtained from FigShare platform following the link https://figshare.com/articles/

COVID-19_severity_correlates_with_airway_epithelium-immune_cell_interactions_identified_by_single-cell_analysis/12436517).

Code availability

All codes used in this work are consistent with the "best practice workflows" suggested by the developers of each used tool as described in the Methods section. RNA-Seq data from different experiments, including the in-house generated sequencing experiments, were analyzed with the same pipeline, based on the described software. Single-cell RNA-Seq experiments, derived from publicly available datasets, were instead processed with the codes defined by the authors, in order to reach consistent cell clustering to be screened for the gene of our interest. Cluster annotation, gene imputation and plots were performed with standard parameters for each of the described tools.

The whole list of custom scripts generated to obtain results shown in Figure 1 are available within FigShare platform with the link: https://figshare.com/articles/software/

Macrophage_expression_and_prognostic_significance_of_the_long_pentraxin_PTX3_in_COVID-19_Custom_scripts_and_analyses/13140248

Further information on research design is available in the Nature Research Reporting Summary linked to this article.

The clinical data that support the findings of this study are available as supplementary raw data and from the corresponding authors upon request.

\section{Field-specific reporting}

Please select the one below that is the best fit for your research. If you are not sure, read the appropriate sections before making your selection.

$\bigotimes$ Life sciences $\quad \square$ Behavioural \& social sciences $\quad \square$ Ecological, evolutionary \& environmental sciences

For a reference copy of the document with all sections, see nature.com/documents/nr-reporting-summary-flat.pdf

\section{Life sciences study design}

All studies must disclose on these points even when the disclosure is negative.

Sample size

This cohort study analyzed a cohort of 96 patients. We included all males and females, 18 years of age or older, admitted to Humanitas Clinical and Research Center (Rozzano, Milan, Italy) between March 4th and May 9, 2020 (data cutoff on May 13th) with a laboratoryconfirmed diagnosis of COVID-19.

A second, independent cohort of 54 patients admitted to the Azienda Socio Sanitaria Territoriale (ASST) Papa Giovanni XXIII, Bergamo, Italy, from February 22nd to April 4th 2020, with a laboratory-confirmed diagnosis of COVID-19, was analyzed to confirm our results.

As this was a pilot observational study, no a-priori sample size calculation was performed. The two samples of patients were used by convenience. Given that the aim of this study was to provide exploratory evidence that PTX3 is associated with death in COVID-19 patients, and we were able to demonstrate this association, a lack of power can be excluded.

Data exclusions We excluded patients younger than18 year old, and pregnant females. Patients with negative assay for SARS-CoV-2 (RT-PCR from pharyngeal swab and bronchoalveolar lavage fluid/endotracheal aspirate) were excluded.

The exclusion criteria were pre-established. Both samples of patients were collected in hospitals not admitting patients less than 18 years old and pregnant women.

Replication

The main results of the clinical part of the paper were replicated (externally validated) in a second independent cohort of patients. 


\section{Reporting for specific materials, systems and methods}

We require information from authors about some types of materials, experimental systems and methods used in many studies. Here, indicate whether each material, system or method listed is relevant to your study. If you are not sure if a list item applies to your research, read the appropriate section before selecting a response.

\begin{tabular}{l|l} 
Materials \& experimental syste \\
\hline $\mathrm{n} / \mathrm{a}$ & Involved in the study \\
\hline & $\bigotimes$ Antibodies \\
\hline & $\square$ Eukaryotic cell lines \\
$\square$ & $\square$ Palaeontology and archaeology \\
$\square$ & $\square$ Clinical data \\
$\square$ & $\square$ Dual use research of concern
\end{tabular}

\begin{tabular}{l|l}
\multicolumn{2}{l}{ Methods } \\
\hline n/a & Involved in the study \\
$\square$ & $\square$ ChIP-seq \\
$\square$ & $\square$ Flow cytometry \\
$\square$ & $\square$ MRI-based neuroimaging
\end{tabular}

\section{Antibodies}

Antibodies used

The following human antibodies were used: anti-CD45-BUV805 (clone HI30, BD Biosciences, Cat. 612891, 1:40); anti-CD11b-BV786
(clone IRCF44, BioLegend, Cat. 301346, 1:40); anti-CD3-BUV737 (clone UCHT1, BD Biosciences, Cat. 564308, 1:80); anti-CD4-PECY5
(clone OKT4, BioLegend, Cat. 317412, 1:40); anti-CD8-BUV496 (clone RPA-T8, BD Biosciences, Cat. 612942, 1:40); anti-CD56-PECF594
(clone NCAM16.2, BD Biosciences, Cat. 564849, 1:20); anti-CD19-APCH7 (clone HIB19, BD Biosciences, Cat. 560727, 1:40); anti-CD14-
AF488 (clone M5E2, BD Biosciences, Cat. 557700, 1:50); anti-CD16-BUV563 (clone 3G8, BD Biosciences, Cat. 748851, 1:20); anti-
CD66b-APCR700 (clone G10F5, BioLegend, Cat. 305114, 1:20); anti-HLA-DR-BV480 (clone G46-6, BD Biosciences, Cat. 566113, 1:40).
Cells were analyzed on FACSymphony (BD Bioscience).
In addition MNB4 and rabbit polyclonal IgG anti human PTX3 produced in-house were used for ELISA and IHC (2 microg/ml final
concentration).

Validation

Antibodies used for FACS analysis are commercially available and validated by the vendors for human cells and for FACS analysis. References are reported for each of them in the manufacturer's website.

The sandwich ELISA is based on the rat monoclonal antibody MNB4, used as capturing antibody, and on biotin-labelled rabbit IgG anti human-PTX3 in detection. Rabbit IgG were purified by immunoaffinity on PTX3-conjugated Sepharose-CNBr columns. Both MNB4 and rabbit IgG were obtained immunizing respectively a rat and rabbits with purified recombinant PTX3 and were selected based on reactivity towards human recombinant PTX3 in ELISA and SDS-PAGE. No cross reaction was observed towards human CRP.

\section{Human research participants}

Policy information about studies involving human research participants

Population characteristics

The study was approved by the Ethical Committee of Humanitas Clinical and Research Center (authorization 233/20), and the requirement for informed consent was waived. Hospital admission criteria were based on a positive assay for SARS-CoV-2 associated with respiratory failure requiring oxygen therapy, or radiological evidence of significant pulmonary infiltrates on chest computed tomography (CT) scan, or reduction in respiratory/cardiopulmonary reserve as assessed by 6 minutes walking test, or due to frailty related with patient comorbidity. We assessed an outcome of death. 52 patients of 96 (54\%) were transferred to ICU because requiring invasive ventilation or non-invasive mechanical ventilation with oxygen fraction over $60 \%$. Patients with continuous positive airway pressure therapy (CPAP) were followed up by ICU outreach team and ward physicians in COVID-19 wards. Acute respiratory syndrome (ARDS) was defined according to the Berlin definition.

The covariate-relevant population characteristics of the participants were age, stay in ICU, SOFA score at admission, and days between symptoms onset and PTX3 test draw, outcome,comorbidities, laboratory tests.

Recruitment

This study analyzed a cohort of 96 consecutive patients admitted to Humanitas Clinical and Research Center (Rozzano, Milan, Italy) between March 4th and May 16rd, 2020 with a laboratory-confirmed diagnosis of COVID-19.

A second, independent cohort of 54 patients admitted to the Azienda Socio Sanitaria Territoriale (ASST) Papa Giovanni XXIII, Bergamo, Italy, from February 22nd to April 4th 2020, with a laboratory-confirmed diagnosis of COVID-19, was analyzed to confirm our results. In this cohort the same criteria of admission of the first cohort had been adopted.

Because of the exclusion criteria applied during the recruitment in both hospitals (i.e. patients less than 18 years old and pregnant women were not included), the results of this study do not apply to these two subpopulations. 
Plots

Confirm that:

\The axis labels state the marker and fluorochrome used (e.g. CD4-FITC).

$\bigotimes$ The axis scales are clearly visible. Include numbers along axes only for bottom left plot of group (a 'group' is an analysis of identical markers).

\All plots are contour plots with outliers or pseudocolor plots.

$\bigotimes$ A numerical value for number of cells or percentage (with statistics) is provided.

\section{Methodology}

Sample preparation

Instrument

Software

Cell population abundance

Gating strategy

凶
Monocytes were isolated from peripheral blood samples of healthy donors and COVID-19 patients. Monocytes were enriched from Ficoll-isolated PBMCs by positive selection using CD14 Microbeads kit (Miltenyi Biotec), according to manufacturer's instructions. Cell viability was determined by Aqua LIVE/Dead Fixable-405nm staining (Invitrogen, Carlsbad, US). Cells were then incubated with a FcR blocking reagent, human (Miltenyi Biotec).

Cells were analyzed on FACSymphony (BD Bioscience).

Data were analyzed with FlowJo software (Treestar, Ashland, US).

Purity of monocytes was $>89 \%$.

The gating strategy was used to verify purity of monocytes used to generate RNA-seq data of Fig. 1b and s shown in Supplementary Figure 1.

All CD45+ cells were gated based on CD45 expression and on the SSC-A morphological parameter to exclude debris. Then, Single Cells were selected on the basis of FSC-A and FSC-H. Dead cells were further excluded through a viability dye. Monocytes were selected as CD11b high CD3- CD19- cells.

$\triangle$ Tick this box to confirm that a figure exemplifying the gating strategy is provided in the Supplementary Information. 